\title{
Contribuições para análise da política de saúde auditiva no Brasil
}

\author{
Contributions for the Brazilian hearing health policy \\ assessment
}

\author{
Maria Cecília Bevilacqua1, Marina Morettin², Tatiana Mendes de Melo ${ }^{3}$, Regina Célia Bortoleto Amantini', \\ Maria Angelina Nardi de Souza Martinez
}

\begin{abstract}
RESUMO
Objetivo: Realizar o levantamento do quantitativo dos procedimentos relacionados à adaptação de aparelho de amplificação sonora individual (AASI) incluídos na Tabela do Sistema Único de Saúde (Tabela SUS). Métodos: Os dados sobre os procedimentos relacionados à adaptação de AASI incluídos na Tabela SUS foram levantados no site www.datasus.gov.br. Após o levantamento desses dados, foi realizada a organização e a análise descritiva da produção dos atendimentos ambulatoriais registrados pelos serviços de saúde auditiva do Brasil, durante o período de novembro de 2004 a julho de 2010. Os dados foram analisados estatisticamente. Resultados: Quanto aos procedimentos relacionados à dispensação de AASI no território nacional no âmbito da saúde auditiva, em 2006, a terapia fonoaudiológica ultrapassou o quantitativo obtido pela adaptação de AASI e, o acompanhamento fonoaudiológico, por sua vez, foi pouco realizado no país. Os AASI com tecnologias B e C vem sendo mais adaptados do que os AASI de tecnologia A e a realização de medida com microfone sonda ou acoplador de 2cc na adaptação dos AASI é pouco realizada em comparação ao ganho funcional. Conclusão: Houve grandes avanços na atenção ao deficiente auditivo no país, mas é necessário aprimorar o acompanhamento dos usuários de AASI, e revisar procedimentos como medidas com microfone sonda e tecnologias dos AASI.
\end{abstract}

Descritores: Audiologia; Perda auditiva; Auxiliares de audição; Sistema Único de Saúde; Políticas públicas de saúde

\section{INTRODUÇÃO}

Na última década no Brasil houve um avanço importante na possibilidade de acesso aos dados do Sistema Único de Saúde (SUS) pela Internet, por meio do Departamento de Informática do SUS - DATASUS (www.datasus.gov.br), órgão da Secretaria Executiva do Ministério da Saúde. Neste sítio é possível levantar informações sobre todos os procedimentos ambulatoriais realizados no país, por meio do Sistema de

Trabalho realizado no Curso de Fonoaudiologia, Faculdade de Odontologia de Bauru, Universidade de São Paulo - USP - Bauru (SP), Brasil.

(1) Curso de Fonoaudiologia, Faculdade de Odontologia de Bauru, Universidade de São Paulo - USP - Bauru (SP), Brasil.

(2) Programa de Pós-graduação (Doutorado) em Epidemiologia, Faculdade de Saúde Pública, Universidade de São Paulo Paulo - USP - São Paulo (SP), Brasil.

(3) Curso de Fonoaudiologia, Universidade de Guarulhos - UnG - Guarulhos (SP), Brasil.

(4) Divisão de Saúde Auditiva do Hospital de Reabilitação de Anomalias Craniofaciais, Universidade de São Paulo - USP - Bauru (SP), Brasil.

(5) Curso de Fonoaudiologia, Pontifícia Universidade Católica de São Paulo - PUC - São Paulo (SP), Brasil.

Endereço para correspondência: Maria Cecília Bevilacqua. R. Silvio Marchione, 3/20, Bauru (SP), Brasil, CEP: 17012-900 (Caixa Postal 1501).

E-mail: cpa@centrinho.usp.br

Recebido em: 22/3/2010; Aceito em: 14/10/2010
Informações Ambulatoriais (SIA/SUS), como parte das políticas de acessibilidade universal do sistema de saúde público brasileiro, estendida também para os dados administrativos.

Há um consenso sobre a importância central do sistema de informação para avaliação das Políticas de saúde, tanto na literatura especializada ${ }^{(1,2)}$, como em relatórios e recomendações de Conferências de Saúde, oficinas de trabalho do SUS e eventos de sociedades científicas. Na área de saúde auditiva, no Brasil, esse tipo de análise foi fundamental para a implementação de uma Política Nacional de Atenção à Saúde Auditiva.

Em 2000, foi estabelecida a primeira Portaria referente à regulamentação, no âmbito ambulatorial, do diagnóstico, da adaptação de aparelhos de amplificação sonora individual (AASI) e do acompanhamento de indivíduos deficientes auditivos (SAS/ MS no 432, publicada em 14 de novembro de 2000). A partir de um levantamento da produção ambulatorial em saúde auditiva registrado no SIA/SUS (DATASUS), do primeiro semestre de 2004, verificou-se que o atendimento ao deficiente auditivo estava fragmentado, sendo que apenas $16 \%$ dos 138 serviços credenciados para realizar esse tipo de atendimento registravam todos os procedimentos relativos à deficiência auditiva da tabela SIA/SUS. Destes serviços, $25 \%$ apresentavam registro de um tipo de procedimento (apenas concessão de AASI ou diagnóstico ou terapia) ${ }^{(3)}$, porém não ofereciam atendimento integral 
conforme os princípios doutrinários do SUS. Apoiado em dados dessa análise, foi verificada a necessidade de reformulação da política de saúde auditiva no país e, em 2004, o Ministério da Saúde, instituiu a Política Nacional de Atenção à Saúde Auditiva através da Portaria GM/MS no 2.073 de 28/09/2004, que contempla desde a promoção da saúde auditiva até a terapia fonoaudiológica, em todos os ciclos da vida.

Passados seis anos da instituição desta nova Política e de consolidação das suas diretrizes, poucos estudos foram realizados com o intuito de analisar os efeitos dessas mudanças nas características de atendimentos dos serviços de saúde auditiva. Tais estudos possibilitariam o exame da implementação dessa política no país e também a otimização dos recursos financeiros disponibilizados na área ${ }^{(4,5)}$.

Nesse sentido, este estudo teve como objetivo realizar o levantamento quantitativo dos procedimentos relacionados à adaptação de AASI incluídos na "Tabela SUS", referentes a dados de todos os serviços de saúde auditiva do país disponibilizados pelo do SIA/SUS do DATASUS.

\section{MÉTODOS}

Para a análise dos resultados, foi realizada primeiramente a seleção dos procedimentos de reabilitação auditiva incluídos na tabela de serviços e classificação do Sistema de Informação Ambulatorial (SIA), códigos e Autorização de Procedimentos de Alta Complexidade (APAC).
Neste trabalho, optou-se por estudar a realização de procedimentos relacionados à adaptação de AASI incluídos na "Tabela SUS", sendo os seguintes procedimentos selecionados: adaptação de AASI, acompanhamento fonoaudiológico, terapia fonoaudiológica, ganho funcional e medidas de ganho de inserção. Os códigos selecionados para análise estão descritos no Quadro 1.

É importante ressaltar que os procedimentos informados não permaneceram inalterados durante todo o período selecionado para estudo. Em novembro de 2008, a tabela de procedimentos do SUS incorporou modificações como alterações na nomenclatura, fato que não constitui grande problema metodológico, pois a diferenciação ocorreu apenas na definição do procedimento (o procedimento, em si, permaneceu inalterado).

Após a escolha dos procedimentos, foi realizado o seu levantamento quantitativo. Esses procedimentos foram aprovados para pagamento pelas Secretarias de Saúde às quais estavam vinculados todos os serviços de saúde auditiva do país. Os dados disponíveis no site www.datasus.gov.br são oriundos do Sistema de Informações Ambulatoriais do SUS - SIA/SUS, gerido pelo Ministério da Saúde, por meio da Secretaria de Assistência à Saúde, em conjunto com as Secretarias Estaduais de Saúde e as Secretarias Municipais de Saúde, processados e disponibilizados pelo DATASUS - Departamento de Informática do SUS, da Secretaria Executiva do Ministério da Saúde.

Após o levantamento desses dados secundários, foi realizada a organização e a análise descritiva da produção dos

Quadro 1. Procedimentos selecionados para análise do presente estudo

\begin{tabular}{|c|c|}
\hline Procedimentos - Até outubro/2007 & Procedimentos - A partir de novembro/2008 \\
\hline 1708224 - Ganho funcional & 0211070033 - Audiometria em campo livre \\
\hline 1708225 - Ganho de inserção & 0211070246 - Pesquisa de ganho de inserção \\
\hline $\begin{array}{l}3901103 \text { - Terapia fonoaudiológica individual em criança } \\
3901104 \text { - Terapia fonoaudiológica individual em adulto }\end{array}$ & 0301070113 - Terapia fonoaudiológica individual \\
\hline $\begin{array}{l}3901105 \text { - Acompanhamento paciente até } 3 \text { anos com AASI uni/ } \\
\text { bilateral } \\
3901106 \text { - Acompanhamento paciente maior de } 3 \text { anos com AASI } \\
\text { uni/bilateral } \\
3901107 \text { - Acompanhamento paciente maior de } 15 \text { anos com AASI } \\
\text { uni/bilateral }\end{array}$ & $\begin{array}{l}0301070032 \text { - Acompanhamento paciente adaptado com AASI uni/ } \\
\text { bilateral }\end{array}$ \\
\hline 3902101 - AASI externo retroauricular tipo A & 0701030127 - AASI externo retroauricular tipo A \\
\hline 3902102 - AASI externo retroauricular tipo B & 0701030135 - AASI externo retroauricular tipo B \\
\hline 3902103 - AASI externo retroauricular tipo C & 0701030143 - AASI externo retroauricular tipo C \\
\hline 3902104 - AASI externo intra-auricular tipo A & 0701030038 - AASI externo intra-auricular tipo A \\
\hline 3902105 - AASI externo intra-auricular tipo B & 0701030046 - AASI externo intra-auricular tipo B \\
\hline 3902106 - AASI externo intra-auricular tipo C & 0701030054 - AASI externo intra-auricular tipo C \\
\hline 3902107 - AASI externo intracanal tipo A & 0701030062 - AASI externo intracanal tipo A \\
\hline 3902108 - AASI externo intracanal tipo B & 0701030070 - AASI externo intracanal tipo B \\
\hline 3902109 - AASI externo intracanal tipo C & 0701030089 - AASI externo intracanal tipo C \\
\hline 3902110 - AASI externo microcanal tipo A & 0701030097 - AASI externo microcanal tipo A \\
\hline 3902111 - AASI externo microcanal tipo B & 0701030100 - AASI externo microcanal tipo B \\
\hline 3902112 - AASI externo microcanal tipo C & $0701030119-$ AASI externo microcanal tipo C \\
\hline 3902113 - AASI externo condução óssea convencional tipo A & 0701030011 - AASI externo condução óssea convencional tipo A \\
\hline 3902114 - AASI externo condução óssea retroauricular tipo A & 0701030020 - AASI externo condução óssea retroauricular tipo A \\
\hline
\end{tabular}


atendimentos ambulatoriais registrados pelos serviços de saúde auditiva, com base na fonte de dados do Ministério da Saúde - item "Informações de Saúde". Como os procedimentos pesquisados pelo estudo são realizados tanto nos domínios de Média como de Alta Complexidade dos serviços de saúde auditiva e como até o ano de 2007 o SIA-SUS não permitia a diferenciação da produção realizada nestes dois níveis de complexidade, optou-se por não fazer análises distintas a partir da variável complexidade do serviço.

Dessa forma, para análise da Política Nacional de Atenção à Saúde Auditiva, na perspectiva da intervenção audiológica, selecionou-se os seguintes parâmetros:

- Distribuição quantitativa dos procedimentos de dispensação de AASI, acompanhamento e terapia fonoaudiológica;

- Distribuição quantitativa dos AASI dispensados, de acordo com a categoria tecnológica A, B ou C, classificados de acordo com o Anexo IV da Portaria 587 (Classificação dos aparelhos de amplificação sonora individuais de acordo com os recursos tecnológicos)(Anexo 1);

- Distribuição dos procedimentos de realização das medidas de ganho de inserção e do ganho funcional para verificação do benefício do dispositivo.

O levantamento incluiu a produção da totalidade da rede existente durante o período de novembro de 2004 a julho de 2010. O número de serviços credenciados ao SUS em todo o território nacional passou de 39 para 143 neste período, como mostra a Tabela 1 .

Foi realizada a análise descritiva, em porcentagem (geral e por ano de produção do atendimento), utilizando o software TABWIN produzido pelo Departamento de Informática do Ministério da Saúde.

\section{RESULTADOS}

Os serviços de saúde auditiva habilitados pelo Ministério da Saúde para a dispensação de AASI devem garantir à pessoa portadora de deficiência auditiva o melhor uso possível do seu resíduo auditivo. Para tanto devem oferecer um processo de reabilitação que garanta não só a seleção e a adaptação do AASI, mas também o acompanhamento periódico para o monitoramento audiológico e a terapia fonoaudiológica. A análise descritiva dos dados quanto à adaptação de AASI, terapia e acompanhamento, de outubro de 2004 a julho de 2010, encontra-se na Tabela 2.

Durante o período analisado foram identificados 2.940 .971 procedimentos, dos quais a maioria refere-se à realização de terapia fonoaudiológica $(61,77 \%)$, ultrapassando a quantidade de adaptações de AASI a partir do ano de 2006 (Tabela 2). Por sua vez, nota-se a produção ambulatorial referente aos atendimentos de acompanhamento reduziu-se ao longo dos anos.

Em relação à categoria tecnológica dos aparelhos auditivos dispensados pelos serviços de saúde auditiva, o Anexo IV da Portaria $\mathrm{n}^{\circ} 587$ estabelece o percentual de adaptação de $50 \%$, $35 \%$ e $15 \%$ para o tipo A, B e C, respectivamente. Durante o período analisado pelo presente estudo 732.113 dispositivos foram fornecidos pelos serviços de saúde auditiva credenciados pelo SUS, sendo $41 \%$ do tipo A, $37 \%$ do tipo B e $22 \%$ do tipo C (Tabela 3).

A análise da categoria tecnológica dispensada em função do ano da produção aponta que durante 2004 e 2005, o percentual de dispensação de AASI a partir da categoria tecnológica está em consonância com o proposto pelo Anexo IV da Portaria $n^{\circ} 587$. Contudo, a partir de 2006 houve redução da dispensação de aparelhos auditivos do tipo A e aumento na adaptação de dispositivos do tipo B e C (Tabela 3).

Após a etapa de seleção e adaptação do aparelho AASI, é imprescindível verificar se os parâmetros prescritos estão em consonância com as necessidades audiológicas apresentadas pelo indivíduo. O Anexo IV da Portaria n 587 estabelece ainda, que a verificação do desempenho e benefício fornecido pelo AASI deve ser realizada utilizando-se medidas em campo livre (ganho funcional) ou medidas com microfone sonda (ganho

Tabela 1. Estabelecimentos de saúde auditiva no Brasil por ano e região em relação à população do país

\begin{tabular}{|c|c|c|c|c|c|c|c|}
\hline Região & 2005 & 2006 & 2007 & 2008 & 2009 & 2010 & População (IBGE) \\
\hline Norte & 0 & 0 & 4 & 5 & 5 & 6 & 14.623 .316 \\
\hline Nordeste & 8 & 5 & 30 & 34 & 35 & 35 & 51.534 .406 \\
\hline Sudeste & 23 & 21 & 51 & 55 & 59 & 59 & 77.873 .120 \\
\hline Sul & 6 & 23 & 33 & 33 & 35 & 34 & 26.733 .595 \\
\hline Centro-oeste & 2 & 2 & 7 & 7 & 9 & 9 & 13.222 .854 \\
\hline Total & 39 & 51 & 125 & 135 & 143 & 143 & 183.987.291 \\
\hline
\end{tabular}

Fonte: DATASUS (www.datasus.gov.br). Setembro de 2010.

IBGE, Contagem da População-2007. Disponível em: http://www.ibge.gov.br/home/estatistica/populacao/contagem2007/contagem_final/tabela1_1.pdf. Agosto 2009.

Tabela 2. Procedimentos realizados nos serviços de Saúde Auditiva no Brasil em relação à intervenção audiológica por ano da produção

\begin{tabular}{|c|c|c|c|c|c|c|c|c|c|c|c|c|c|c|c|c|}
\hline & \multicolumn{2}{|c|}{$2004^{*}$} & \multicolumn{2}{|c|}{2005} & \multicolumn{2}{|c|}{2006} & \multicolumn{2}{|c|}{2007} & \multicolumn{2}{|c|}{2008} & \multicolumn{2}{|c|}{2009} & \multicolumn{2}{|c|}{2010} & \multicolumn{2}{|c|}{ Total } \\
\hline & $n$ & $\%$ & $\mathrm{n}$ & $\%$ & $\mathrm{n}$ & $\%$ & $\mathrm{n}$ & $\%$ & $\mathrm{n}$ & $\%$ & $\mathrm{n}$ & $\%$ & $n$ & $\%$ & $\mathrm{n}$ & $\%$ \\
\hline Terapia & 3605 & 15,00 & 78.151 & 33,00 & 142.369 & 47,91 & 212.655 & 53,27 & 475.511 & 67,48 & 550.415 & 70,40 & 353.974 & 71,00 & 1.816 .680 & 61,77 \\
\hline Acompanhamento & 8176 & 34,00 & 48.775 & 20,64 & 54.832 & 18,45 & 59.834 & 15,00 & 77.460 & 11,00 & 88.334 & 11,30 & 54.767 & 11,00 & 392.178 & 13,33 \\
\hline Dispensação AASI & 12.259 & 51,00 & 109.359 & 46,36 & 99.942 & 33,64 & 126.671 & 31,73 & 151.665 & 21,52 & 143.004 & 18,30 & 89.213 & 18,00 & 732.113 & 24,09 \\
\hline Total & 24.040 & 100,00 & 236.285 & 100,00 & 297.143 & 100,00 & 399.160 & 100,00 & 704.636 & 100,00 & 781.753 & 100,00 & 497.954 & 100,00 & 2.940 .971 & 100,00 \\
\hline
\end{tabular}


Tabela 3. Percentual de dispensação de AASI de acordo com a categoria tecnológica, em função do ano de produção do procedimento (2004 a julho/2010)

\begin{tabular}{|c|c|c|c|c|c|c|c|c|}
\hline \multirow{3}{*}{ Ano } & \multicolumn{8}{|c|}{ Categoria tecnológica } \\
\hline & \multicolumn{2}{|c|}{$A$} & \multicolumn{2}{|c|}{$B$} & \multicolumn{2}{|c|}{$\mathrm{C}$} & \multicolumn{2}{|c|}{ Total } \\
\hline & $n$ & $\%$ & $n$ & $\%$ & $n$ & $\%$ & $n$ & $\%$ \\
\hline 2004 & 5970 & 48,69 & 4377 & 35,70 & 1912 & 15,61 & 12.259 & 100,00 \\
\hline 2005 & 53.049 & 48,50 & 39.256 & 35,89 & 17.054 & 15,61 & 109.359 & 100,00 \\
\hline 2006 & 44.178 & 44,20 & 37.071 & 33,00 & 18.693 & 22,80 & 99.942 & 100,00 \\
\hline 2007 & 54.800 & 43,26 & 45.320 & 35,77 & 26.551 & 20,97 & 126.671 & 100,00 \\
\hline 2008 & 57.370 & 37,82 & 57.225 & 37,73 & 37.070 & 24,45 & 151.665 & 100,00 \\
\hline 2009 & 51.750 & 36,18 & 55.109 & 38,53 & 36.145 & 25,29 & 143.004 & 100,00 \\
\hline 2010 & 32.856 & 36,82 & 33.191 & 37,20 & 23.166 & 25,98 & 89.213 & 100,00 \\
\hline Total & 299.973 & 40,97 & 271.549 & 37,09 & 160.591 & 21,94 & 732.113 & 100,00 \\
\hline
\end{tabular}

de inserção), ressaltando-se que com crianças menores de três anos deve ser necessariamente utilizada esta última forma de verificação mencionada.

A Figura 1 apresenta, em porcentagem, a quantidade de procedimentos referentes às medidas com microfone sonda (ganho de inserção) e as medidas em campo livre (ganho funcional), entre 2004 a julho/2009. Por sua vez, a Tabela 4 apresenta a análise descritiva desta apreciação, em função do ano de realização dos procedimentos.

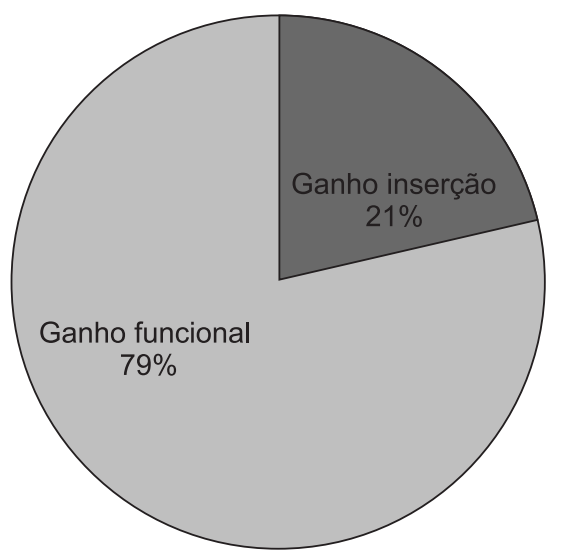

Figura 1. Análise descritiva do quantitativo de procedimentos referentes às medidas com microfone sonda e as medidas em campo livre, entre 2004 a julho/2010

Tabela 4. Análise descritiva referente às medidas com microfone sonda e medidas em campo livre, em função do ano de realização dos procedimentos (2004 a julho/2010)

\begin{tabular}{lcccccc}
\hline \multirow{2}{*}{ Ano } & \multicolumn{2}{c}{ Ganho inserção } & \multicolumn{2}{c}{ Ganho funcional } & \multicolumn{2}{c}{ Total } \\
\cline { 2 - 7 } & $\mathrm{n}$ & $\%$ & $\mathrm{n}$ & $\%$ & $\mathrm{n}$ & $\%$ \\
\hline 2004 & 2222 & 26,70 & 6097 & 73,30 & 8319 & 100,00 \\
2005 & 1869 & 3,76 & 47.798 & 96,24 & 49.667 & 100,00 \\
2006 & 1529 & 2,36 & 63.203 & 97,64 & 64.732 & 100,00 \\
2007 & 3590 & 4,13 & 83.351 & 95,87 & 86.941 & 100,00 \\
2008 & 51.499 & 30,19 & 119.086 & 69,81 & 170.585 & 100,00 \\
2009 & 50.420 & 27,60 & 132.281 & 72,40 & 182.701 & 100,00 \\
2010 & 34.508 & 28,20 & 87.836 & 71,80 & 122.344 & 100,00 \\
\hline Total & 145637 & 21,25 & 539.652 & 78,75 & 685.289 & 100,00 \\
\hline
\end{tabular}

\section{DISCUSSÃO}

Informações oriundas da produção ambulatorial dos serviços de saúde são de grande valia para subsidiar a tomada de decisões ${ }^{(6,7)}$. Áreas que envolvem tecnologias, como no atendimento ao deficiente auditivo, necessitam de estudos que utilizem tais informações para a avaliação da política e a efetividade do tratamento, permitindo otimizar a verba pública $^{(8-10)}$.

Embora não seja usual o aproveitamento de dados secundários, como os utilizados no presente estudo, ${ }^{(11)}$ os mesmos podem ser muito úteis, pois nos bancos de dados nacionais, como o SIA/SUS, são cadastradas diversas informações referentes à produção ambulatorial de todos os serviços de saúde credenciados pelo Ministério da Saúde ${ }^{(12,13)}$. Tal registro possibilita analisar aspectos importantes da Política Nacional de Atenção à Saúde Auditiva (PNASA) ao longo de seu processo de implantação.

Desde a publicação do banco, em 2004, o Brasil tem sido citado internacionalmente como modelo na área. É sabido que a consolidação desta política será um processo dinâmico de construção, uma vez que em tudo o que se inicia, faz-se necessário o aprimoramento. Cabe, então, aos profissionais de saúde uma reflexão que leve à identificação de novas necessidades e à busca de soluções para as mesmas.

Na literatura científica, alguns estudos foram desenvolvidos em serviços de saúde auditiva do SUS. Embora estes trabalhos estejam amplamente focados em aspectos de relevância científica para a Audiologia, a contribuição dos mesmos para um maior entendimento sobre a dinâmica desta Política tem sido restrita, fato que mereceria reflexão aprofundada. Assim, a literatura científica é escassa, quando relacionada às políticas públicas voltadas à saúde auditiva, caracterizada por trabalhos pontuais $^{(4,5)}$.

Além disso, a contribuição de experiências internacionais é limitada, devido às diferenças dos serviços e demais especificidades da Política de Atenção à Saúde Auditiva de cada país ${ }^{(14-16)}$. No Brasil, todo o processo de reabilitação auditiva oferecido pelo Ministério da Saúde é gratuíto, enquanto que em outros países, sistemas de reembolso, descontos e subsidios parciais são as formas mais usadas na aquisição dos AASI oferecida pelos governos. 
O que se almeja neste trabalho é descrever a quantidade de procedimentos realizados pelos serviços credenciados pelo SUS desde a implantação da PNASA, a fim de identificar possíveis dificuldades e sugerir modificações para que os serviços possam atender da melhor forma possível os pressupostos preconizados pela mesma.

Até o período analisado neste estudo, julho/2010, 143 serviços foram habilitados pelo Ministério da Saúde nas categorias Média e Alta Complexidade ambulatorial em saúde auditiva. Segundo o parâmetro estabelecido na Portaria SAS/ MS n ${ }^{\circ}$ 587/04 para implantação dos serviços especializados, de uma unidade para cada 1,5 milhões de habitantes, estava previsto um total de 126 unidades no país, mas por decisão dos gestores estaduais e municipais, o número de unidades habilitadas ultrapassou esse número, podendo atingir 165 unidades em todo o país. Dessa maneira, até o momento, $86 \%$ da implantação da rede foi atingida ${ }^{(3)}$.

Nas regiões Norte e Centro-Oeste o número de serviços de saúde auditiva credenciados é bastante inferior quando comparado a outras regiões, fato que restringe a universalidade do acesso dos pacientes com perda de audição no país. Além disso, a busca pela assistência em serviços de outras regiões poderá acarretar um aumento do custo do tratamento por parte dos órgãos governamentais, como por exemplo, em relação ao transporte, já que é oferecido pelo governo um auxílio para Tratamento Fora do Domicílio (TFD).

O desafio após a habilitação de todos os serviços previstos é a organização da rede de referência e contra-referência, para otimizar o funcionamento de todo o sistema. Em alguns estados, nos quais o número de serviços habilitados atingiu $100 \%$, a organização da rede de referência e contra-referência é feita pelos Departamentos Regionais de Saúde (DRS), como no estado de São Paulo, que regulariza o encaminhamento do paciente para o serviço, de acordo com a residência. Assim, o paciente deverá ser encaminhado, por sua DRS, para o serviço de referência localizado em sua região, consolidando uma rede efetiva de referência e contra-referência para o atendimento integral ao deficiente auditivo ${ }^{(17)}$, como ocorre em países desenvolvidos ${ }^{(18)}$.

A fragmentação do atendimento na rede de saúde auditiva do SUS antes da implantação da PNASA, com predomínio do atendimento centrado na dispensação de AASI, restringiu a realização de outros procedimentos como a terapia e o acompanhamento, o que é criticável já que tais procedimentos são reconhecidos como imprescindíveis para que os usuários de AASI de fato tenham benefício com o uso dos AASI ${ }^{(3)}$.

Após 2004, ao considerar a terapia fonoaudiológica essencial para que o paciente seja beneficiado com o uso da amplificação, verifica-se que este procedimento vem sendo incorporado gradativamente à rotina (Tabela 2). A incorporação desse encaminhamento reflete a tendência mundial nos serviços de saúde auditiva ${ }^{(19,20)}$. A partir de 2006, a produção ambulatorial referente à terapia fonoaudiológica ultrapassou $\mathrm{o}$ número atingido pela adaptação de AASI e acompanhamento fonoaudiológico, o que pode sugerir uma reorganização dos serviços para a realização deste procedimento e maior capacitação dos profissionais para compreender a importância da terapia dentro do processo de reabilitação auditiva.
Porém, este procedimento ainda está muito aquém do esperado, quando se considera a proporção de terapia fonoaudiológica por AASI dispensado e o número mínimo de quatro sessões de terapia fonoaudiológica, definido pela Portaria de Saúde para população adulta. A realização desta etapa da intervenção audiológica é de fundamental importância, uma vez que há consenso dos profissionais da área sobre a indicação dos AASI, para fornecer a maior quantidade de informação acústica possível embora sejam limitados seus recursos para modificação direta do processamento ou do comportamento do usuário ${ }^{(21,22)}$.

Por sua vez, nota-se que a produção ambulatorial referente aos atendimentos de acompanhamento reduziu-se ao longo dos anos (Tabela 2), o que é uma tendência contrária ao esperado. Em grande parte dos pacientes com perda auditiva, a adaptação dos AASIs é feita bilateralmente, o que poderia justificar a diferença quantitativa entre a dispensação do dispositivo e o acompanhamento audiológico, pois o procedimento de acompanhamento é registrado uma única vez ao ano, independentemente da orelha adaptada (monoaural ou binaural). Contudo, de acordo com o Anexo IV da Portaria n ${ }^{\circ} 587$, o acompanhamento refere-se à realização de reavaliações audiológicas e dos AASIs para ajustes anualmente e, deste modo, seria esperado maior número destes procedimentos.

Este resultado nos leva à seguinte questão: os serviços não estão realizando o acompanhamento fonoaudiológico ou há uma falta de adesão dos pacientes ao processo de reabilitação auditiva. Nesse último caso, podem ser cogitadas a falta de esclarecimento sobre o fato de que a adaptação do AASI é somente uma etapa do processo ou a dificuldade de acesso ao serviço (nos casos de pacientes que vão em busca do serviço em outras regiões do país ${ }^{(17)}$. A regulação de vagas e o teto estabelecido para os serviços de saúde auditiva, pelos gestores também deve ser cogitada, pois há tendência a priorizar o atendimento aos usuários com deficiência auditiva que buscam o serviço pela primeira vez e que ainda não tiveram acesso ao AASI.

Ao levantar a segunda hipótese como verdadeira, ressaltase mais uma vez a importância da atenção à saúde auditiva, em todos os níveis da rede. Neste ponto ressalta-se que a atuação dos profissionais na atenção básica na saúde auditiva, pode ocorrer não só na promoção da saúde auditiva, na identificação de indivíduos com possíveis desordens da função auditiva, como também na orientação da família, quanto à existência de tratamento e à necessidade de continuidade do mesmo, o que auxiliará a garantir a adesão da família a todas as etapas da intervenção audiológica ${ }^{(23)}$.

Com relação à adaptação de AASI, é possível observar que a partir de 2006 os AASI de categoria tecnológica A, B e C fornecidos pelo SUS indicam tendência que não corresponde ao previsto no Anexo IV da Portaria n ${ }^{\circ} 587$. A partir de 2006 houve uma redução da dispensação de AASI do tipo A e aumento na adaptação de dispositivos do tipo B e C (Tabela 3), corroborando recomendações internacionais sobre a adaptação de AASI com recursos mais sofisticados, como por exemplo, o algoritmo para redução de ruído, para melhor desempenho do paciente em situações de vida diária ${ }^{(24)}$, especialmente na população infantil ${ }^{(25,26)}$. 
Dados referentes a estudos epidemiológicos a respeito da deficiência auditiva no Brasil com base populacional ${ }^{(27,28)}$ evidenciam a predominância de perdas auditivas de grau leve e moderada, que necessitam de aparelhos auditivos mais flexíveis (como o tipo B e C), reforçando assim a necessidade de revisão imediata da Portaria quanto às categorias tecnológicas.

É necessário também considerar o avanço tecnológico dos AASI, uma vez que os de tipo A já são obsoletos, e isto é fundamental para continuar assegurando a dispensação dos dispositivos pelo SUS com qualidade, otimizando os recursos financeiros alocados para a rede de saúde auditiva.

Apesar de a medida com microfone sonda (ganho de inserção) ser descrita na literatura como procedimento preferencial na verificação do desempenho e benefício fornecido pelo AASI, no Brasil, as medidas em campo livre (ganho funcional) ainda são realizadas de forma predominante nos serviços de saúde auditiva do SUS (Figura 3 e Tabela 4). O predomínio da avaliação comportamental do desempenho de tecnologias não lineares, como é o caso dos aparelhos do tipo B e C, também são contrários ao recomendado por protocolos internacionais, uma vez que o ganho funcional não fornece qualquer informação sobre a compressão utilizada e o desempenho para sons de diferentes níveis de entrada ${ }^{(25,26)}$.

Este achado ainda é mais alarmante se considerarmos a adaptação pediátrica, ao passo que a prescrição da amplificação em bebês deve utilizar métodos objetivos como o RECD - diferença entre o ouvido real e o acoplador de $2 \mathrm{cc}^{(25,26)}$.

Tal fato não pode ser justificado pela ausência do equipamento de microfone sonda nos serviços de Média e Alta Complexidade, uma vez que a existência deste equipamento é obrigatória para o credenciamento. Neste sentido, a falta de treinamento profissional para a realização deste procedimento parece ser fator relevante.

Torna-se necessário o aperfeiçoamento dos profissionais que atuam nesses serviços para a realização das medidas com microfone sonda ${ }^{(29)}$, a fim de garantir a realização de procedimentos que proporcionem melhor adaptação do AASI e, consequentemente, maior benefício ao paciente.

Devemos ressaltar, ainda, limitações do estudo, como por exemplo, a mudança na "Tabela do SUS" em 2008, o que inviabilizou verificar o número de procedimentos de terapia fonoaudiológica de forma separada entre adultos e crianças. A análise deste procedimento em função da idade do paciente poderia contribuir para a apreciação mais aprofundada dos dados apresentados neste trabalho, uma vez que o foco nesta etapa da intervenção audiológica apresenta, objetivos distintos para estas populações-alvo.

Os dados sencundários, como os provenientes do banco de dados do Ministério da Saúde, devem ser analisados com cautela, uma vez que os mesmos estão mais susceptíveis a erros de digitação e inconsistências ${ }^{(30)}$, podendo interferir na confiabilidade das informações obtidas. No entanto, este tipo de limitação é inerente a estudos de qualquer área da saúde que utilizam as informações provenientes do SIA-SUS. Desta forma, os dados referentes à produção ambulatorial deste banco de dados não devem ser desconsiderados na análise da Política de Atenção à Saúde Auditiva, pois são de fácil acesso e disponibilizam informações de serviços de todo o país.

\section{CONCLUSÃO}

A análise do número de procedimentos relacionados à dispensação de AASI incluídos na tabela de serviços disponibilizados no DATASUS permitiu mostrar avanços importantes no país, no que se refere à saúde auditiva, após o estabelecimento da Política Nacional de Atenção à Saúde Auditiva, assim como sugerir aprimoramentos referentes ao estabelecimento de algumas diretrizes e ao funcionamento dos serviços.

Os resultados mostraram que houve um avanço em relação à cobertura nacional dos serviços de saúde auditiva, com $86 \%$ da rede já implantada em todo o país, em 2010. Nas regiões Norte e Centro-Oeste a cobertura está mais defasada em relação às outras regiões.

Quanto aos procedimentos relacionados à dispensação de AASI no território nacional no âmbito da saúde auditiva, a terapia fonoaudiológica ultrapassou o número atingido pela adaptação de AASI. O acompanhamento fonoaudiológico, por sua vez, ainda é pouco realizado, sendo necessário que os serviços se organizem para investigar quais os motivos para a não realização de acompanhamento dos pacientes adaptados com AASI no país.

Quanto à tecnologia dos AASI, desde 2006, aqueles com tecnologias $\mathrm{B}$ e $\mathrm{C}$ vem sendo mais adaptados do que os AASI de tecnologia A. Esse fato mostra a necessidade de atualização da classificação dos tipos de aparelhos quanto à tecnologia por parte do governo federal. Além disso, a realização das medidas com microfone sonda ou acoplador de 2cc na adaptação dos AASI na rotina de atendimento dos serviços de saúde auditiva, ainda se encontram muito aquém do esperado. 


\begin{abstract}
Purpose: To quantity the procedures related to hearing aid fitting included in Hearing Health Care National Policy (Tabela SUS). Methods: Data about fitting procedures included in Tabela SUS were gathered in the website www.datasus.gov.br. After data collection, it was conducted the organization and descriptive analysis of the production of ambulatory consults registered by hearing health services in Brazil, during the period from November 2004 to July 2010. Data were statistically analyzed. Results: Regarding procedures related to dispensing hearing aids in national territory, in 2006, speech-language therapy exceeded the amount obtained by hearing aid fitting, and audiological follow-up was little frequent. Hearing aids with technologies B and C have been adapted more than hearing aids with technology $\mathrm{A}$, and the use of measures with a real-ear measurement or $2 \mathrm{cc}$ coupler in the adaptation of hearing aids is uncommon, in comparison to functional gain measures. Conclusion: There has been great progresses in the area of hearing impaired attention in the country, but it is necessary to improve the audiological follow-up to hearing aid users, and to review procedures such as the real-ear measurement and technologies of hearing aids.
\end{abstract}

Keywords: Audiology; Hearing loss; Hearing aids; Unified Health System; Health public police

\title{
REFERÊNCIAS
}

1. Carvalho AI, Bodstein RC, Hartz Z, Matida AH. Concepts and approaches in the evaluation of health promotion. Ciênc Saúde Coletiva. 2004;9(3):521-9.

2. Bosi ML, Uchimura KY. Avaliação da qualidade ou avaliação qualitativa do cuidado em saúde? Rev Saúde Pública 2007;41(1):150-3.

3. Daher CV, Pisaneschi E. A política nacional de atenção à saúde auditiva: a atenção especializada às pessoas com deficiência auditiva no SUS. In: Bevilacqua MC, Martinez MA, Balleri AS, Pupo AC, Reis AC, Frota SM, organizadores. Saúde auditiva no Brasil: políticas, serviços e sistemas. São José dos Campos: Pulso; 2010. p.15-30.

4. Teixeira CF. Estudo avaliativo da política de atenção à saúde auditiva: estudo de caso em Pernambuco - Recife [tese]. Recife (PE): Fundação Oswaldo Cruz, Centro de Pesquisas Ageu Magalhães; 2007.

5. Amaral LS. A política nacional de atenção à saúde auditiva: avanços e entraves no município do Rio de Janeiro [dissertação]. Rio de Janeiro: Escola Nacional de Saúde Pública Sergio Arouca; 2009.

6. Taiul L. A importância do uso dos sistemas de informação em estudos e pesquisas na área de epidemiologia [editorial]. Epidemiol Serv Saúde. 2004;13(1):5-6.

7. Barros MB. A importância dos sistemas de informação e dos inquéritos de base populacional para avaliações de saúde [editorial]. Epidemiol Serv Saúde. 2004;13(4):199-200.

8. Bevilacqua MC, Melo TM, Morettin M, Lopes AC. A avaliação de serviços em audiologia: concepções e perspectivas. Rev Soc Bras Fonoaudiol. 2009;14(3):421-6.

9. Clark JL. Hearing loss in Mozambique: current data from Inhambane Province. Int J Audiol. 2008;47 Suppl 1:S49-56.

10. Chao TK, Chen TH. Cost-effectiveness of hearing aids in the hearing-impaired elderly: a probabilistic approach. Otol Neurotol. 2008;29(6):776-83.

11. Pinheiro RS, Camargo Junior KR, Coeli CM. Relacionamento de bases de dados em saúde. Cad Saúde Colet (Rio J). 2006;14(2):195-6.

12. Buss PM. Assistência hospitalar no Brasil (1984-1991): uma análise preliminar baseada no SIH/SUS. Inf Epidemiol SUS. 1993;2(2):5-12.

13. Travassos C. O sistema de informações hospitalares do Sistema Único de Saúde; SIH-SUS. Rio de Janeiro: FIOCRUZ; 1996 [mimeo].

14. Madriz JJ. Audiology in Latin America: hearing impairment, resources and services. Scand Audiol Suppl. 2001;(53):85-92.

15. Barto G, Davis A, Mair LWS, Parving A, Rosenhall U, Sorri M. Provision of hearing aid services: a comparison between the Nordic countries and the United Kingdom. Scand Audiol Suppl. 2001;(54):1620.

16. Hadjikakou K, Petridou L, Stylianou C. Evaluation of the support services provided to deaf children attending secondary general schools in Cyprus. J Deaf Stud Deaf Educ. 2005;10(2):203-11.
17. Morettin M. Avaliação dos benefícios e satisfação dos usuários de aparelhos de amplificação sonora individuais nos serviços de audiologia do SUS [dissertação]. São Paulo: Universidade de São Paulo, Faculdade de Saúde Pública; 2008.

18. Eley KA, Fitzgerald JE. Quality improvement in action. Direct general practitioner referrals to audiology for the provision of hearing aids: a single centre review. Qual Prim Care. 2010;18(3):201-6.

19. Smith JL, Mitchell P, Wang JJ, Leeder SR. A health policy for hearing impairment in older Australians: what should it include? Aust New Zealand Health Policy. 2005;2:31.

20. Pratt SR. Barriers to hearing health care: current status and a glimpse at the future. Am J Audiol. 2010;19(1):1-2.

21. Hawkins DB. Effectiveness of counseling-based adult group aural rehabilitation programs: a systematic review of the evidence. J Am Acad Audiol. 2005;16(7):485-93.

22. Boothroyd A. Adult aural rehabilitation: what is it and does it work? Trends Amplif. 2007;11(2):63-71.

23. Alvarenga KF, Bevilacqua MC, Martinez MA, Melo TM, Blasca WQ, Taga MF. Proposta para capacitação de agentes comunitários de saúde em saúde auditiva. Pró-Fono. 2008;20(3):171-6.

24. Krishnamurti S. Audiology service models in a family physician practice setting. Perspectives on Audiology. 2010;6(1):24-32.

25. Bagatto M, Scollie SD, Hyde M, Seewald R. Protocol for the provision of amplification within the Ontario infant hearing program. Int J Audiol. 2010;49 Suppl 1:S70-9.

26. King AM. The national protocol for paediatric amplification in Australia. Int J Audiol. 2010;49 Suppl 1:S64-9.

27. Béria JU, Raymann BC, Gigante LP, Figueiredo AC, Jotz G, Roithman R, Selaimen da Costa S, Garcez V, Scherer C, Smith A. Hearing impairment and socioeconomic factors: a population-based survey of an urban locality in southern Brazil. Rev Panam Salud Publica. 2007;21(6):381-7.

28. Bevilacqua MC. Levantamento das alterações auditivas da população urbana de Monte Negro - Rondônia/Brasil. Bauru: Universidade de São Paulo, Faculdade de Odontologia de Bauru; 2007. Relatório Final: Processo CNPq no 479793/2004-2.

29. Swanepoel de W, Clark JL, Koekemoer D, Hall JW 3rd, Krumm M, Ferrari DV, McPherson B, Olusanya BO, Mars M, Russo I, Barajas JJ. Telehealth in audiology: the need and potential to reach underserved communities. Int J Audiol. 2010;49(3):195-202.

30. Couto RD, Latorre MR, Di Santi SM, Natal D. Malária autóctone notificada no Estado de São Paulo: aspectos clínicos e epidemiológicos de 1980 a 2007. Rev Soc Bras Med Trop. 2010;43(1):52-8. 
Anexo 1. Classificação dos aparelhos de amplificação sonora individuais de acordo com os recursos tecnológicos

\begin{tabular}{|c|c|c|c|}
\hline & \multicolumn{3}{|c|}{ Tecnologia } \\
\hline & Tipo A & Tiро B & Tipo C \\
\hline Programação & Programáveis ou não & Programáveis ou não & Programáveis \\
\hline Modo de condução do som & Aérea ou óssea & Aérea & Aérea \\
\hline Controle de saída & $\begin{array}{l}\text { Corte de picos ou Compressão } \\
\text { de limitação }\end{array}$ & Compressão de limitação & Compressão de limitação \\
\hline Compressão & Monocanal & WDRC mono ou multicanal & WDRC multicanal \\
\hline Controles disponíveis & $\begin{array}{c}\text { Ganho, corte de graves e/ou } \\
\text { corte de agudos, controle para } \\
\text { saída máxima. }\end{array}$ & $\begin{array}{l}\text { Ganho, corte de graves e/ou } \\
\text { corte de agudos, controle para } \\
\text { saída máxima, controle do limiar } \\
\text { e/ou razão de compressão. }\end{array}$ & $\begin{array}{l}\text { Ganho, corte de graves e/ou } \\
\text { corte de agudos, controle para } \\
\text { saída máxima, controle do limiar } \\
\text { e/ou razão de compressão e/ } \\
\text { ou controle das constantes de } \\
\text { tempo da compressão. }\end{array}$ \\
\hline Controle de volume & Manual & $\begin{array}{c}\text { Manual } \\
\text { e/ou automático }\end{array}$ & Manual e ou automático \\
\hline Entradas alternativas & $\begin{array}{l}\text { Bobina telefônica e/ou entrada } \\
\text { de audio* }\end{array}$ & $\begin{array}{l}\text { Bobina telefônica e/ou entrada } \\
\text { de áudio* }\end{array}$ & $\begin{array}{c}\text { Bobina telefônica e ou entrada } \\
\text { de áudio* }\end{array}$ \\
\hline Memórias & Única & Única ou multimemória & Única ou multimemória \\
\hline Microfone & Omnidirecional ou direcional & Omnidirecional ou direcional & Omnidirecional ou direcional \\
\hline Controle de ruído & & & Algoritmo para redução de ruído \\
\hline Expansão & & & Expansão \\
\hline Microfonia & & & $\begin{array}{l}\text { Algoritmo para redução de } \\
\text { microfonia }\end{array}$ \\
\hline
\end{tabular}

Fonte: Portaria SAS 587 de outubro de 2004 - Item 5, Anexo IV. 